\title{
The Design Strategy of High-Density Primary Schools in Shenzhen
}

\author{
Yanli Sun* \\ South China University of Technology, Guangzhou 510000, Guangdong Province, China \\ *Corresponding author: Yanli Sun, a1394433463@163.com \\ Copyright: () 2022 Author(s). This is an open-access article distributed under the terms of the Creative Commons Attribution License (CC \\ BY 4.0), permitting distribution and reproduction in any medium, provided the original work is cited.

\begin{abstract}
The construction of high-density primary schools in Shenzhen is facing new challenges. Therefore, it is essential to look for ways to meet the demand for degrees, realize education and teaching reform, eliminate the shackles of design code, and adapt to the local climate. From the perspective of design methodology, this article discusses the design strategy of Shenzhen's high-density primary school campus from four aspects: compact layout, multiple functions, open space, and personalized design, aiming to provide ideas for the construction of primary school campuses.
\end{abstract}

Keywords: High-density primary schools; Urban primary and secondary schools; Campus layout; Design research

Online publication: January 12, 2022

\section{Introduction}

With the rapid increase of urban population and shortage of land resources, there has been a sky-high demand for degrees in Shenzhen. It has been foretold that in the future, there will be an increase of 740,000 degrees. In order to break through this dilemma, the construction of high-density primary schools is a foregone conclusion. In 2018, the Shenzhen Municipal Bureau of Planning and Natural Resources launched an event - "Towards a New Campus: Futian New Campus Action Plan," whereby emerging domestic and foreign architects attended the event and advised on Shenzhen's high-density campus. Up to now, the New Campus Action Plan has lasted for three seasons. A large number of high-quality campus practices have landed in Shenzhen, providing a new model for the southern high-density campus ${ }^{[1]}$. Beginning with the influencing factors of the design of high-density primary schools, this paper discusses the design strategy from multiple perspectives through case analysis and comparison.

\section{Factors influencing the design of high-density primary schools}

\subsection{High-density urban environment}

Since the reform and opening-up along with the acceleration of China's urbanization process, Shenzhen has seen a dramatic increase in its population, and it has become the most densely populated city in China. In addition to the implementation of the three-child policy in 2021 and the schooling needs of the children of local migrant workers, school-age children are continuously being enrolled. The lack of planning in the initial stage of the city has resulted in scarce land resources for the establishment of schools. Shenzhen General Primary and Secondary School Construction Standard Guidelines (2016) stipulated that the perstudent land area has been reduced by $16 \%$ to $40 \%$ compared to the 2007 guidelines ${ }^{[2]}$. Even more, the 2016 to year "standard" has not kept up with the actual situation in Shenzhen. For example, in the new 
campus action plan implemented from 2018 to 2021, the land for the construction of high-density primary schools cannot meet the standards of the 2016 guidelines. This reflects a direct contradiction between the huge degree gap and the shortage of land resources. Under the pressure of high-density urban environment, primary schools are passively seeking a breakthrough in design through encryption and upgrading.

\subsection{New educational concept}

With the development of the times, traditional talents who are proficient in reading, writing, and arithmetic can no longer meet the needs of today's innovation-oriented society. Correspondingly, the traditional concept of competence education and its guidance of the "class-based teaching system" model cannot adapt to today's teaching needs. Affected by foreign open teaching concepts, China's teaching concepts have also breezed into a "post-competence education period" with broader meaning and more modern characteristics [3]. During this period, talents with professional capabilities and complex relationships are regarded as the needs of the society; in addition, the class-based, exploratory teaching model is also widely accepted. As the substance support for education, campus buildings should be based on ideas, uphold people-oriented design, and build a new class of campus that truly promotes the development of education. As a predicament of China's reform and opening up, the construction of high-density primary schools should be guided by new educational concepts to build a more open, free, and flexible "school of the future."

\subsection{Design code constraints}

In the high-density dispute, the contradiction between the innovative campus spatial pattern and the normative indicators was the first to be stimulated. Among the harsh land conditions, the national school building code, which was born in the low-density era during the early stages of urbanization, did not take into account the practicality and timeliness of the code and could not be fully applicable to high-density elementary school campuses. The main influencing factors include regulations on sports fields, sunshine, noise, and spatial paradigms. These factors are entangled with each other, which to a certain extent leads to the shackles of the spatial pattern, making domestic schools present a rigid and forced spatial layout ${ }^{[4]}$. In the long run, students in such a solidified environment will inevitably face rigid thinking, which is inconsistent with the demand for innovative talents by the country. In order to see a breakthrough, the "new campus plan" puts forward a need to transform rigid norms to flexible mechanisms and rigid indicators to dynamic problem solving. It aims to flexibly interpret and apply norms based on people's needs as well as help high-density primary school campuses resolve contradictions and innovate effectively ${ }^{[1]}$.

\subsection{Climate adaptability}

Shenzhen is located in southern China and the south-central coastal area of Guangdong Province. It has a typical south subtropical monsoon climate, with long summers, short winters, a mild climate, abundant sunshine, and abundant rainfall. Therefore, for Shenzhen, which is rich in sunshine resources in low latitude areas, shading and ventilation are important aspects which need to be considered in the design, compared with the 25 meters spacing control of sunshine in the specification. In addition, playgrounds with overhead shelters should also be widely used supplementary to campus activity space. In summary, when designing campus buildings, appropriate ventilation, moisture-proof, shading, and heat insulation strategies should be considered. Actively adopting architectural design techniques and passively adopting green building technologies are also involved. The combination of the two forms a campus with the characteristics of Shenzhen ${ }^{[2]}$. 


\section{Research on the design strategy of high-density primary schools}

In harsh land environment, the quality of campus construction is likely to be affected, but the spatial relevance and vitality improve. In the design of a high-density primary school campus, balancing the area pressure, maximizing the use of space while improving the quality of the campus has become the focus in the design strategy. Therefore, this article summarizes the design strategy of high-density primary schools in Shenzhen from four aspects, which are compact layout, multiple functions, open space, and personalized design, through case comparison (Figure 1).

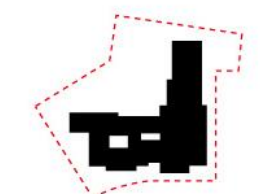

Meili Primary School

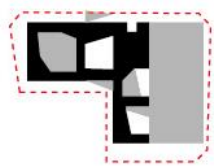

Shangxing Primary School

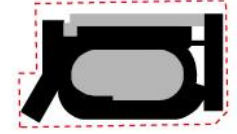

Aihua Primary School

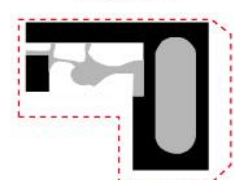

Qiaodong Primary School-1

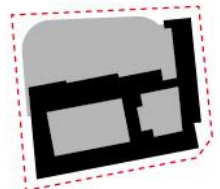

Xinzhou Primary School

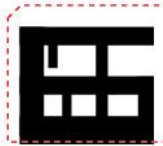

Shixia Primary

School

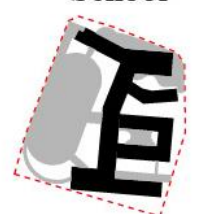

Liyuan Primary School-1

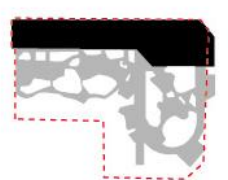

Qiaodong Primary School-2

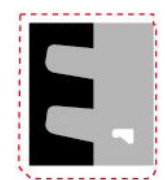

Hongling Primary School

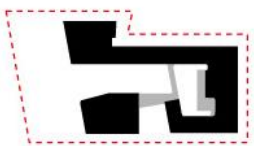

Liwan Primary School

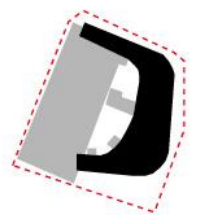

Liyuan Primary School-2

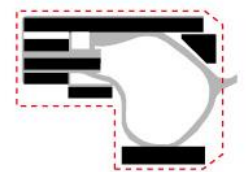

Qiaodong Primary School-3

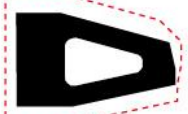

Lianhua Primary School

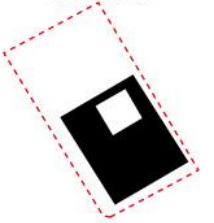

Liyuan Primary School

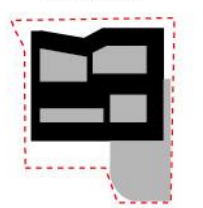

Meihong Primary School-2

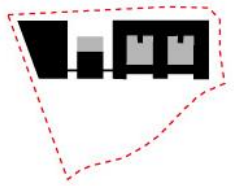

Haixi Primary

School

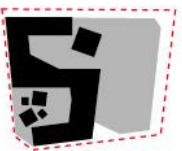

Xinsha Primary

School

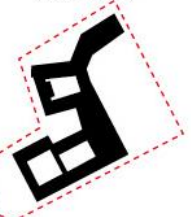

Longhua Foreign

Language School

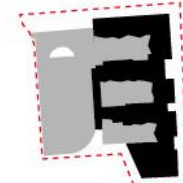

Meihong Primary School-3

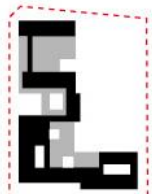

Haian Primary

School

Figure 1. Layout of high-density primary schools in Shenzhen (self-drawn by the author)

\subsection{Compact layout}

In order to save and make full use of land resources, high-density primary schools, which have a compact layout, differ from traditional low-density campuses. Compact layout is further manifested as high density in the horizontal direction and three-dimensional in the vertical direction.

In the horizontal direction, the building density of a high-density campus is allowed to a certain degree of freedom, up to $61 \%$, and problems such as retreat lines can be adjusted through a series of design measures. In the design process of Xinsha Primary School, the campus proposed the sharing of arcade street; hence, the Planning Bureau agreed to the special approval of "zero retreat line along the street for the large platform and 3 meters retreat line along the exterior wall of the street." On the one hand, more construction land and activity space have been obtained; on the other hand, the negative relationship between campus boundary and urban isolation has been solved creatively. Through the selected large platform, the building did not only prevent school safety problems, such as rock climbing, but also provide a public pedestrian 
corridor for the city ${ }^{[5]}$. In addition, the 25 meters sunlight spacing and noise spacing have also been flexibly resolved through clever layout and the use of technology, further achieving a compact campus layout in the horizontal direction.

In the vertical direction, for efficient use of space, the three-dimensional layout has become an effective solution strategy. In traditional campus design, the sports field occupies the largest area and has the greatest impact on the campus layout. However, in high-density primary school campus design, a certain degree of freedom in elevation is allowed. Sports fields can be elevated to the middle layer or the roof layer, among which the typical cases of elevation to the middle layer include Hongling Primary School, Xinzhou Primary School, and Xinsha Primary School, while those that reached the roof layer include Shenzhen Lotus Primary School, Aihua Primary School, and Qiaodong Primary School (Figure 2). The elevation of sports field has greatly increased the construction area of the campus and improved the space utilization within limited land.

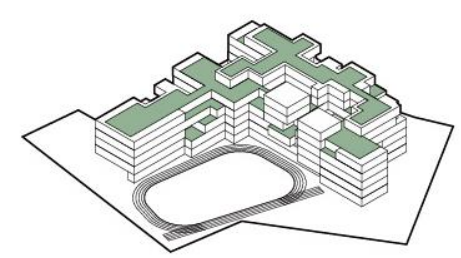

Meili primary school

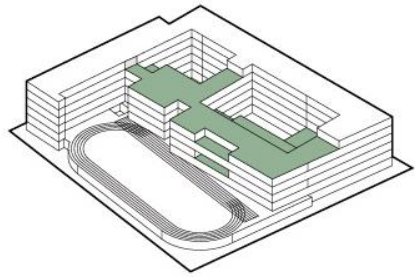

Xinzhou primary school

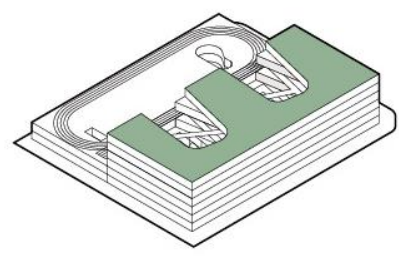

Hongling primary school

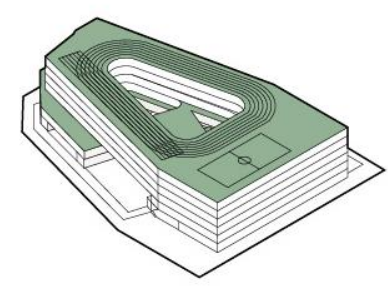

Lianhua primary school

Figure 2. Four typical cases (self-drawn by the author)

In addition, the campus layout does not only extend upwards, but also downwards to the underground space. The underground space is fully utilized; combined with the ground floor plan, it can effectively accommodate the large public space in the school building, such as swimming pools, indoor courts, public lecture halls, co-class classrooms, libraries, teaching spaces for calligraphy, dance, and other special skills, service kitchens, restaurants, etc. In designing Shenzhen Hongling Primary School's underground space (Figure 2), the underground space is connected with the gentle slope of the first-floor overhead layer, and the rich design techniques, such as high-altitude, create a large number of open and comfortable semiindoor activity spaces. Softened with green slopes, its borders are combined with the overhead ground floor and the inner courtyard of the building. In that way, the underground space is no longer a dark and humid area but has become a new "playground" that is bright and airy. The second or third floor can be used as an underground parking lot and combined with the campus shuttle area to relieve the pressure of the city [6]. The basement in Meili Primary School (Figure 2) has been merged with the parking lot, and a sunken plaza space has been established as a pick-up area. This is a good solution to relieve students' space pressure of going up and down the school entrance.

The roof terrace of the campus can also be effectively used as a farm or outdoor activity space to provide students with diversified activity venues. The regulations stipulate that the main teaching rooms need to be set up on the fourth floor or below. In high-density primary schools, in order to realize the vertical layout of the campus, the specifications are flexibly interpreted, special classrooms are arranged on the fifth floor, and administrative office rooms are arranged on the sixth floor.

\subsection{Multiple functions}

The compounding of functions refers to the juxtaposition and interleaving of two or even multiple functions in the same building space to form a compounding spatial layout. The composite of functions here also has 
two meanings: one is the composite of functions, and the other is the composite of time.

In order to accommodate various types of activities in the same space, the space should be used effectively. In high-density primary schools, the shortage of space urges architects to make full use of open spaces, such as platforms and corridors, as places for students' recess activities. The free layout and open boundaries of these spaces allow them to be expanded into multifunctional and multi-scene spaces.

Student activities in primary schools have a strong periodicity according to the curriculum arrangement. In traditional campuses, a large area of space is not frequently used as a large-scale activity place. Therefore, this kind of space is considered to be used staggered in time in high-density primary schools. For example, lecture halls can be used for co-class classrooms; restaurants and other places can also be used as activity venues; other than that, basketball courts can be used as badminton and volleyball courts. In addition to the compound inside the campus, the compound can also be realized between the campus and the community, thus reflecting the concept of community sharing. During winter and summer vacations, the public facilities in elementary schools are often vacant, which is such a waste. Shared campus service facilities can share resources with the community during this period. On the one hand, it provides more convenient services to urban citizens; on the other hand, it prevents the wastage of resources.

\subsection{Open space}

In the traditional sense, the single teaching mode has undergone diversified changes. At the same time, it needs to rely on open space as a support. An open space can effectively accommodate various activities under the condition of fuzzy boundaries, free form, and flexible functions. On the one hand, it can be used as an informal learning space to provide support for the reform of teaching mode from "teaching" to "learning"; on the other hand, it can be used as a place for communication and games. Professor Aoki, who has been engaged in primary and secondary school research for a long time, has shown in a study that games and play activities between classes are beneficial to students' emotional mediation, mental rejuvenation, as well as physical and mental health ${ }^{[7]}$.

The design of Xinzhou Primary School (Figure 2) makes full use of the scattered layout of the roof platform to create a multiscale open activity platform for students, which solves the contradiction between the ten-minute limited rest time and the long distance from the ground space ${ }^{[8]}$. In the design process of Meili Primary School, the architects drew inspiration from the village in the city, imitated the spatial form of the village, broadened the traditional 1.8 meters corridor to 6 meters every two floors, as well as created a two-story "Book Street" activity space. Not only does this effectively buffer interclass activities, but it can also be used as an open learning space to provide students with a reading place and create an open, free, as well as flexible scholarly campus.

\subsection{Personalized design}

He Jianxiang, the designer of Hongling Primary School, said, "A school is not just a single unit, a house, or even a space, but a place for young people to grow for a long period of time." The high density of the campus itself is questionable as there is squeezing of activity space, and its design is also more likely to breed mediocre or even unreasonable architectural layouts due to limited land resources. The depressive high-density environment will also affect young people and children. In the design of high-density schools, more attention should be paid to the creation of space personality and vitality to alleviate the impact of unfavorable environmental factors.

The People's Primary School, designed by Zhixiang Architectural Design, retains a "small forest" at the original site. Although the students' activity space is squeezed to a certain extent, natural elements such as sunshine, wind, and four seasons can be more vividly perceived here, and these will become specific and 
beautiful memories in students' childhood learning and life. From the perspective of children, the designers have created a unique entrance and podium platform for the main playground in Xinsha Primary School. The red castle, mountain roads, lane spaces, rooftop farms, triangular hills, and other areas will inevitably become children's entertainment and play-heaven ${ }^{[5]}$. Shixia Primary School uses the big trees surrounding the urban courtyard to shape the memory of the place ${ }^{[9]}$.

As Duan Yifu said in Space and Place: An Empirical Perspective, "Place means safety, and space means freedom. We all hope to have both safety and freedom. Space and place are the basic components of the world we live in, so we are accustomed to it." Architects need to regain their childhood memories and create truly personalized and distinctive school buildings.

\section{Conclusion}

With the strengthening of China's comprehensive national power and the urgent need for innovative talents, the establishment of high-density primary schools in Shenzhen has expanded from a "case" to a "paradigm." In order to cope with the increasingly severe urban high-density environment, adapt to new teaching models, eliminate the shackles of design code, and cope with the local climate, the design strategy of high-density primary schools can be summarized as compact layout, multiple functions, open space, and personalized design. Among them, compact layout refers to high density in the horizontal direction and threedimensional in the vertical direction; multiple functions refer to the compounding of functions in space and time; open space refers to the free and flexible arrangement of space for open activities; personalized design refers to the strengthening of campus characteristics and students' needs.

\section{Acknowledgements}

I would like to thank Dongyi architecture and WAU for the information provided for this article.

\section{Disclosure statement}

The author declares that there is no conflict of interest.

\section{References}

[1] Zhou H, 2021, From Initiation to Action: Retrospect and Reflection on the Mechanism Innovation of "Futian New Campus Action Plan". Architecture Journal, 629(3): 1-9.

[2] Su X, 2019, Research on the Adaptive Design Strategy of the Architectural Environment of Shenzhen Primary and Secondary Schools. Shenzhen University.

[3] Li L, 2016, A Preliminary Study on the Architectural Design of Domestic Primary and Secondary Schools Under the Current Educational Concept. Tianjin University.

[4] Zhao J, 2016, Study on the Possibility of Primary and Secondary School Architectural Design Under the Constraint of Regulations. Zhejiang University.

[5] Xie J, Zhou Z, 2021, Xinsha Primary School: A Campus Design That Returns to the Perspective of Children. Architecture Journal, 629(3): 35-41.

[6] He J, 2020, From Household Furnace to Urban Sky - Shenzhen Hongling Experimental Primary School Campus Design Notes. Architecture Journal, 2020(1): 32-36.

[7] Li Z, 2000, A New Type of Primary and Secondary School Building Form Adapted to Competence Education - New Type of Building Space in Primary and Secondary Schools. Journal of Xi'an University of Architecture and Technology (Natural Science Edition), 2000(3): 237-241. 
[8] Xiao Y, Zou Y, Xiao Y, 2021, Stacked Garden Building a High-density City Between Class Paradise Design Considerations for Xinzhou Primary School in Futian District. Architecture Journal, 629(3): 27-34.

[9] Wang W, 2021, The Rising Tree - Seven Steps Towards the Urban Courtyard Campus. Architecture Journal, 629(3): 19-26.

\section{Publisher's note}

Bio-Byword Scientific Publishing remains neutral with regard to jurisdictional claims in published maps and institutional affiliations. 